\section{Lead Selenide Cells for Infra-Red Spectroscopy}

THE advantages of lead sulphide cells ${ }^{1}$ as detectors in infra-red spectrometers have already been described $^{2}$ for the region between 1 and $3 \mu$. Although it appears that the useful range of these cells may be extended slightly by working at liquid air and liquid hydrogen temperatures ${ }^{3}$, it seemed to us more profitable to explore further the possibilities of lead selenide cells as a means of extending the range of photoconductive methods in the infra-red. Reports of war-time work in Germany indicated that lead selenide had a peak sensitivity near $3 \cdot 4 \mu$ as com. pared with the lead sulphide peak near $2 \cdot 6 \mu$; but no figures have been available on the actual performance of these German cells, relative to some standard such as a good vacuum thermocouple. The fact that lead selenide cells never got beyond the laboratory stage in Germany would seem to indicate that progress there had not reached the stage where the lead selenide cell was superior to the thermocouple or bolometer.

We have recently made lead selenide cells by the method outlined by Simpson ${ }^{4}$ which are superior to a Hilger-Schwarz thermocouple, at least as far as $4 \mu$, and have a time of response which is well below $50 \times 10^{-6}$ sec. These have been tested in a prism spectrometer (lithium fluoride prism) of high resolving power against a first-rate Hilger-Schwarz vacuum thermocouple as standard. By comparing the slit widths necessary to give the same signal/noise ratio when the thermocouple has a 'Pyrex' window in front, of comparable thickness to the base of the lead selenide cell, we obtained the sensitivity curve shown in the graph. It will be observed that the lead selenide cell has two maxima of sensitivity, one near $1 \cdot 6 \mu$ and the other near $3 \cdot 3 \mu$, the former being considerably the higher of the two. The factors controlling the relative heights of these two maxima have not yet been elucidated.

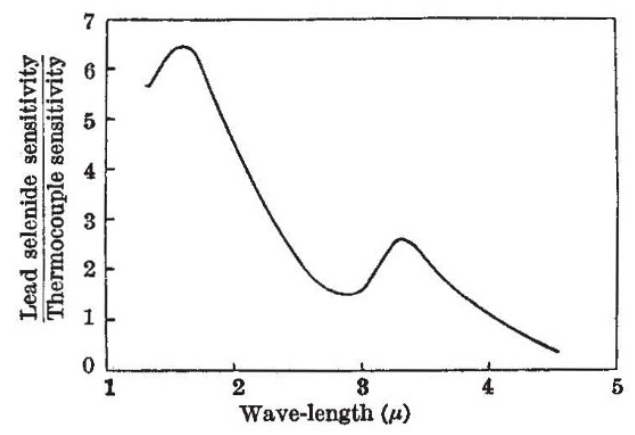

Although the lead selenide cells so far made are inferior in performance to lead sulphide cells (comparing absolute sensitivities at their respective maxima) we have no reason to believe that the optimum method of preparation and usage has yet been achieved. These preliminary results are, however, most encouraging, and even if no further improvement is obtained the present lead selenide cells are an important advance on the lead sulphide cell. The extension of the usable range from $3 \mu$ to $4 \mu$ means that virtually all absorption bands due to the stretching vibrations of hydrogen atoms can be examined with a much higher resolving power and at much greater speed than was previously possible.
The 'Pyrex' envelope now used is admittedly a drawback in that its absorption limits the effective range to $3.4 \mu$, but we have found that a quartz envelope gives an improvement, and it is hoped that a suitable window can be arranged for the base of the envelope to enable the full range to be exploited.

We are indebted to the Admiralty and to the Telecommunications Research Establishment at Malvern for the loan of equipment used in making and testing these cells.

\section{E. BlackWELL \\ O. Simpson \\ G. B. B. M. Sutherland}

Cavendish and Colloid Science Laboratories, Cambridge.

Aug. 15.

${ }^{1}$ Sosnowski, L., Starkiewicz, J., and Simpson, O.. Nature, 158, 28 (1946), and 159, $818(1947)$. 2 Sutherland, G. B. B. M., Blackwell, D. E., and Fellgett, P. B.,
Nature, 158, 873 (1946).

${ }^{3}$ Moss, T. S., Nature, 159, 476 (1947).

4 Simpson, O., Nature, see preceding communication.

\section{Interaction Terms in Molecular Vibrations}

We have recently been investigating the magnitudes and signs of the interaction terms in the potential functions of triatomic molecules $X Y Z$. These arise from interactions between two bond-lengths ( $X Y$ and $Y Z$ ), or between one bond-length and the angle $X Y Z$. In our study of these terms we have distinguished three distinct effects : (1) resonance effects ; (2) interaction between non-bonded atoms (here $X$ and $Z$ ); (3) changes of hybridization at the central atom (here $Y$ ). A proper understanding of all three of these is necessary before even the signs of the interaction terms can be adequately explained.

An example of each of these three effects may be given.

(1) The resonance effect is shown in the linear molecule $\mathrm{N}-\mathrm{N}-\mathrm{O}$, where the chief resonating structures are I and II,

$$
\overline{\mathrm{N}}=\underset{\text { (I) }}{+}=0 \quad \underset{\text { (II) }}{\mathrm{N}}=\stackrel{+}{\mathrm{N}}-\overline{\mathrm{O}}
$$

and the wave function is

$$
\psi=\psi_{\mathrm{I}}+\lambda \psi_{\mathrm{Ir}} .
$$

If, now, the $\mathrm{N}-\mathrm{O}$ bond-length is increased (by $\left.\Delta r_{1}\right)$, $\psi_{\text {II }}$ is favoured energetically, so that $\lambda$ is increased and the structure II acquires greater importance. This means, as has been mentioned elsewhere ${ }^{1}$, a shortening of the $\mathrm{N}-\mathrm{N}$ bond (by $\Delta r_{2}$ ) and consequently a positive $f_{12}$ in the potential energy expression for linear vibrations :

$$
2 V=f_{1} \overline{\Delta r}_{1}^{2}+f_{2}{\overline{\Delta r_{2}}}^{2}+2 f_{12} \Delta r_{1} \Delta r_{2} .
$$

This sign for $f_{12}$ agrees with the vibration spectrum ${ }^{1}$.

(2) The interaction between non-bonded atoms is shown in the linear molecule $\mathrm{H}-\mathrm{C} \equiv \mathrm{N}$. Here the dipole moment for both bonds is directed in the same way $(+\rightarrow)$, so that $H$ is positively charged and $\mathrm{N}$ is negatively charged. There is, therefore, a strong attraction between them. It can be shown that this leads to a negative value of $f_{12}$, in agreement with experiment ${ }^{2}$.

(3) The changes in hybridization at the central atom are illustrated by the water molecule $\mathrm{H}-\mathrm{O}-\mathrm{H}$. 